Supporting information for:

\title{
Two-fluorophore mobile phone imaging of biplexed real-time NAATs overcomes optical artifacts in highly scattering porous media
}

Kamal G. Shah, Sujatha Kumar, Vidhi Singh, Louise Hansen, Erin Heiniger, Joshua D. Bishop, Barry Lutz, and Paul Yager

Department of Bioengineering, University of Washington, Seattle, Washington, 98195.

Table of Contents

Figure S1

Page 2

Figure S2

Page 4

Figure S3

Page 5

Figure S4

Page 5 

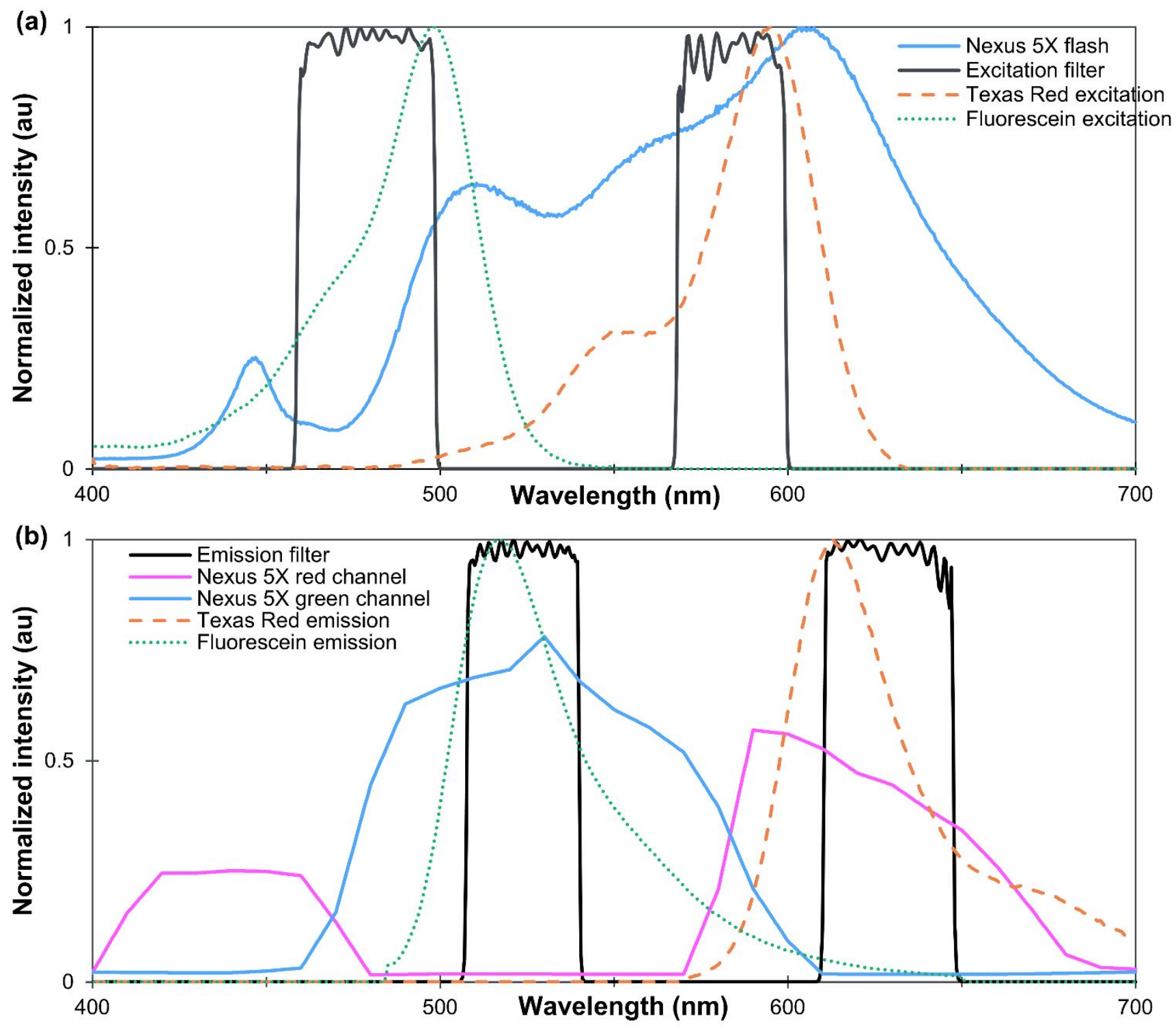


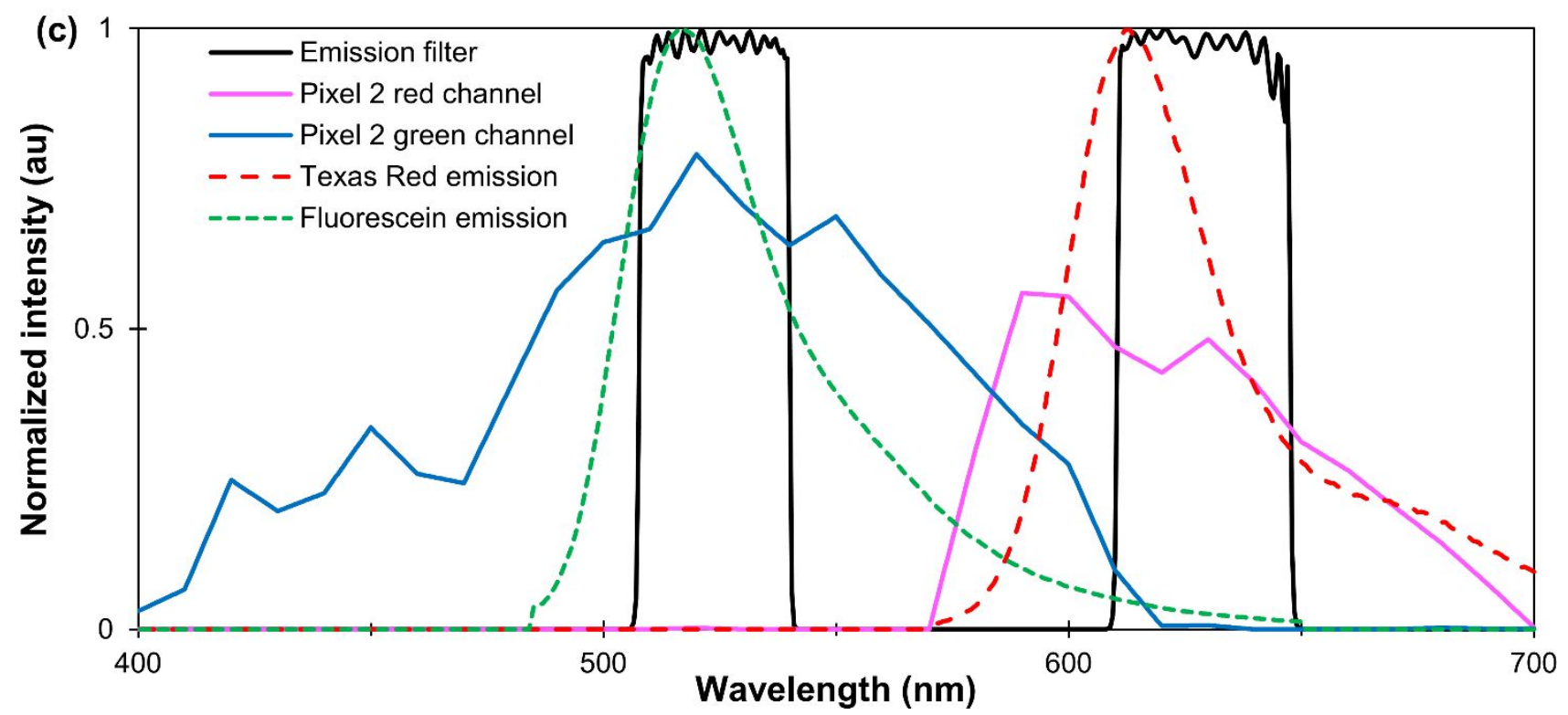

Supplemental Figure S1: Principle of operation of the dual fluorophore mobile phone reader. (a) The multipass excitation filter (solid black curve) transmits wavelengths of the mobile phone flash (solid blue curve) that correspond to the excitation spectra of fluorescein (dotted green curve) and Texas Red (dashed red curve). (b, c) The multi-pass emission filter (solid black curve) transmits wavelengths of the fluorescein (dotted green curve) and Texas Red (dashed red curve) emission that correspond to the green (solid blue curve) and red channels (solid orange curve) of photos taken by the mobile phone, respectively. Photos taken with the flash thereby image fluorescein and Texas Red. Shown are the specific flash and camera spectra of the Nexus 5X mobile phone (a, b), along with the camera spectra of the Pixel 2 mobile phone (c). Filter spectra are adapted from Semrock, and the fluorophore spectra are from ThermoFisher. Phone spectra were measured with the approach outlined in Shah et al., Analytical Chemistry, 2018. 
(a)

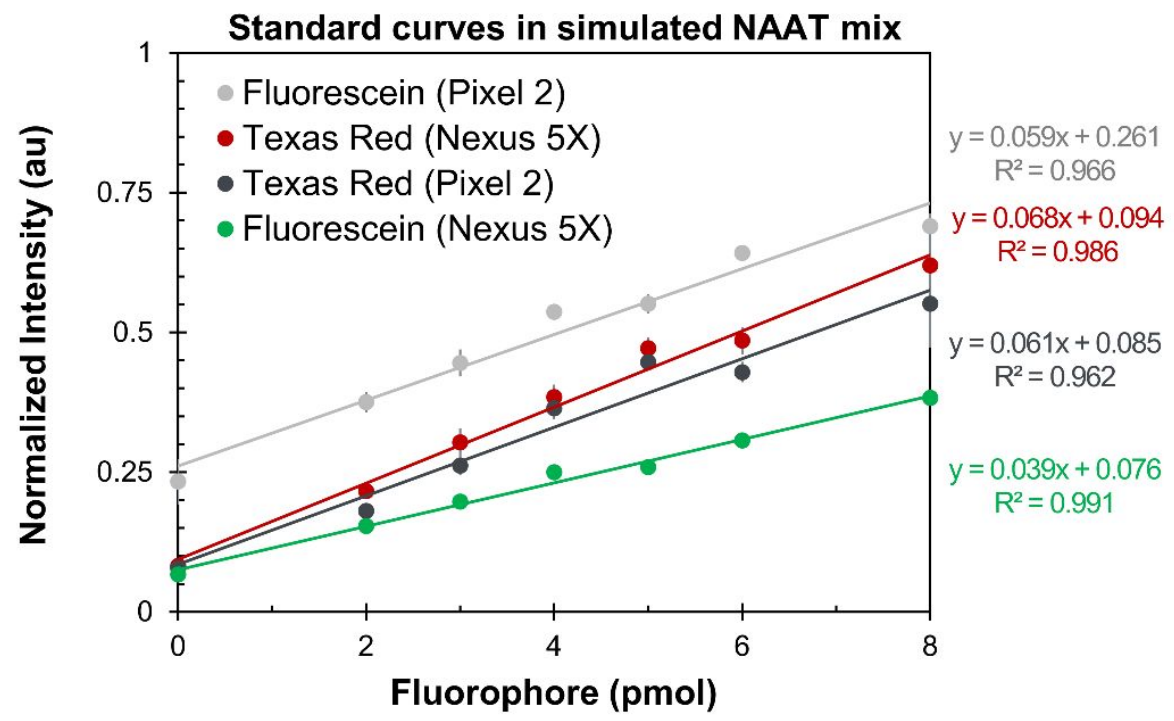

(b)

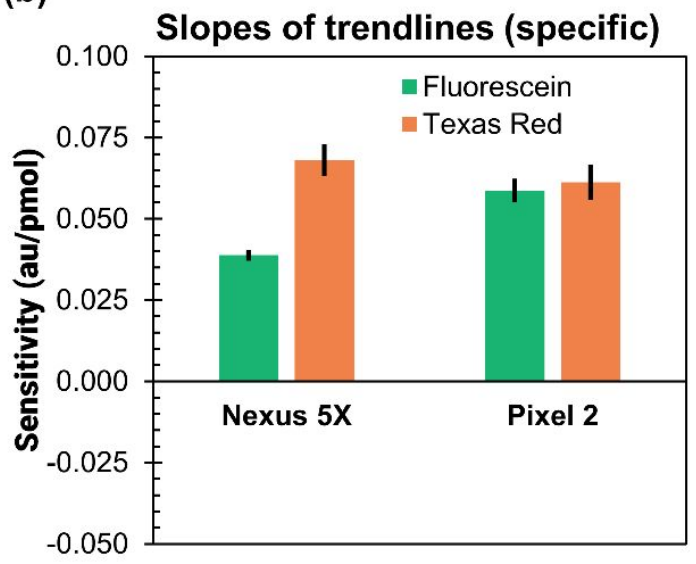

(c)

Crosstalk

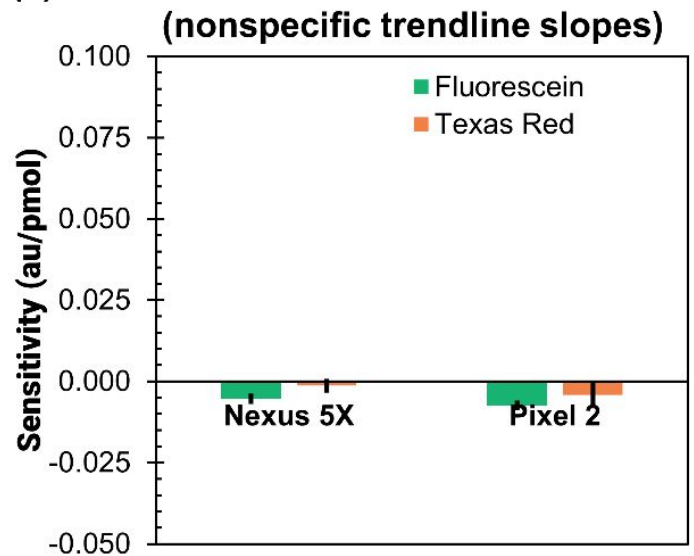

Supplemental Figure S2: (a) Standard curves of glass fiber pads containing fluorescein or Texas Red imaged by the mobile phone fluorescence reader. Pads were saturated with mock nucleic acid amplification reagent mixtures that mimic the $\mathrm{pH}$, salt, and viscosity of iSDA and imaged at iSDA temperatures. Limits of detection for both fluorophores are lower than the nominal fluorescent probe amounts $(4.0 \mathrm{pmol})$ in each iSDA reaction (Nexus 5X: $1.2 \mathrm{pmol}$ (Texas Red) and $0.64 \mathrm{pmol}$ (fluorescein) and Pixel 2: $1.7 \mathrm{pmol}$ (Texas Red) and $3.7 \mathrm{pmol}$ (fluorescein)). Points indicate mean and standard error of the mean $(\mathrm{n}=4)$. (b) Both phones were similarly sensitive to fluorescein and Texas Red (as indicated by the slope of the linear regression of fluorescein in the green channel and Texas Red in the red channel, $\mathrm{p}>0.7)$. (c) There was minimal crosstalk as suggested by the near-zero slope in the nonspecific color channels (fluorescein in the red color channel and Texas Red in the green color channel). Bars indicate the slope and error bars indicate the standard error of the slope estimates. 
(a) Photos

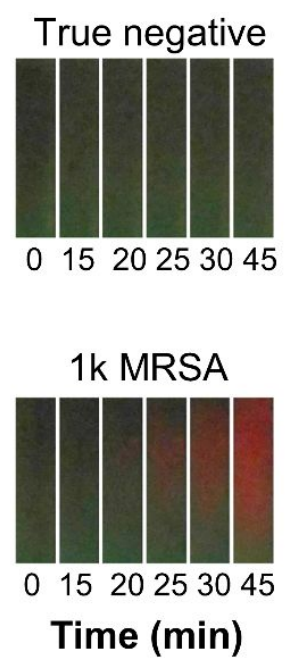

(b) Real-time amplification curves

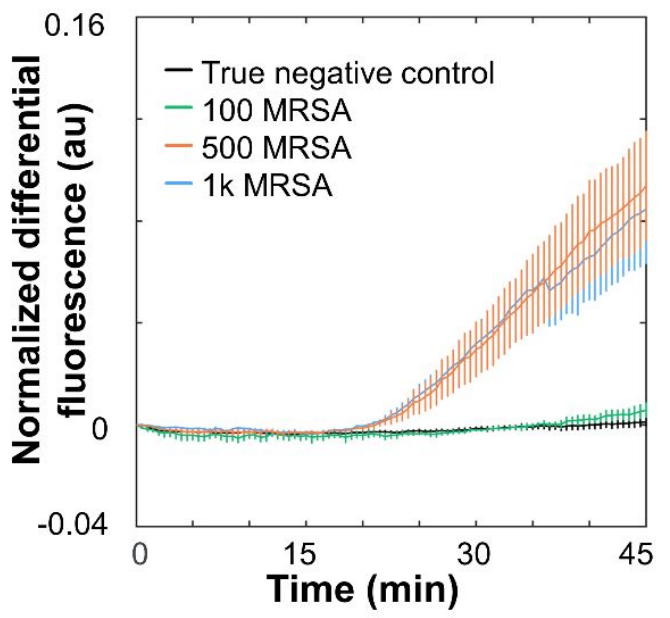

(c) Endpoint differential fluorescence

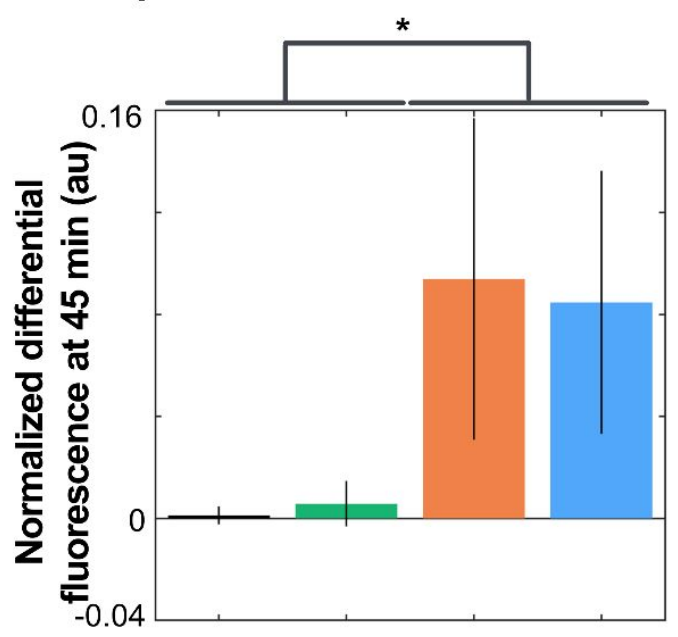

Supplemental Figure S3: Imaging singleplexed amplification reactions in the MD NAAT in real time with the Nexus 5X phone. Glass fiber pads containing lyophilized isothermal strand displacement amplification reagents (with a red-emitting fluorescent probe for mec $A$ ) were rehydrated with samples containing MRSA genomic DNA or water. Pads were heated in the MD NAAT for 45 minutes and imaged about every 30 seconds with a Nexus 5X equipped with multipass excitation and emission filters for fluorescein and Texas Red. (a) Photos of amplifying pads show substantial red fluorescence in a MRSA-containing pad. (b) Realtime curves show the average differential fluorescence intensity across each pad (red color channel minus green channel) over time. The curves show rapid amplification of 500 and 1000 copies of MRSA. Lines show mean and standard error of the mean $(n=3)$. (c) The endpoint differential fluorescence at 45 minutes is significantly higher in pads containing at least 500 copies of MRSA than in the negative control $\left({ }^{*} \mathrm{p}<0.001\right.$, one-way ANOVA). Bars show mean and error bars show 95\% confidence intervals.
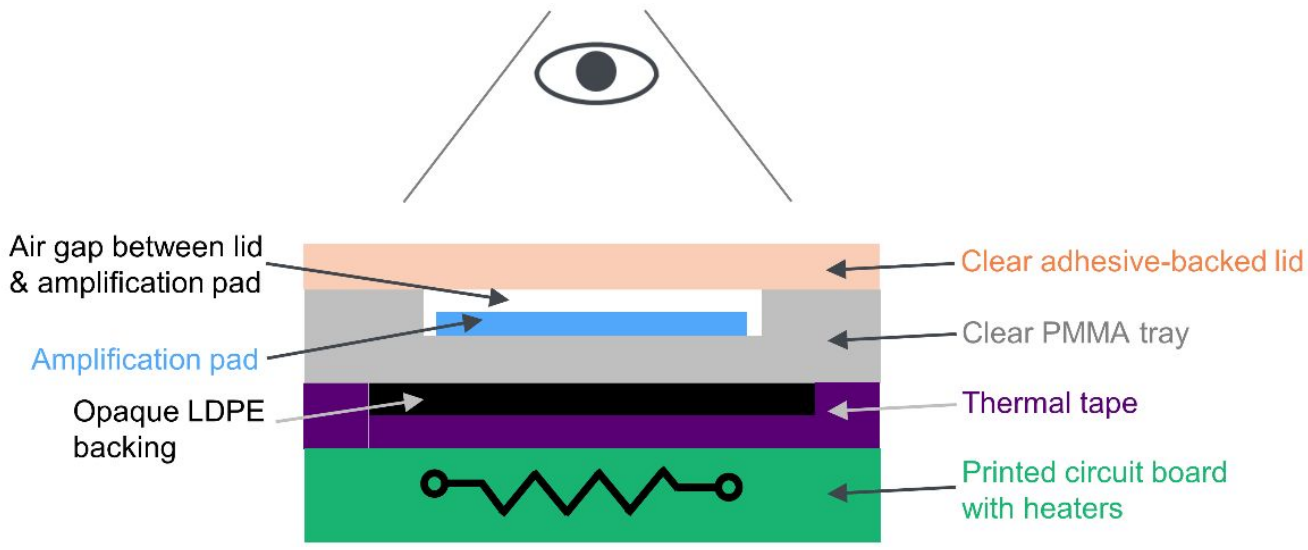

Supplemental Figure S4: Cross-section of the amplification zone during imaging illustrates how condensation could have lowered fluorescence intensities in acquired images. The amplification pads (in blue) were heated by a printed circuit board solely from below. Evaporated liquid from the amplification pads could have condensed on the clear adhesive-backed lid above the amplification zone (shown in orange) due to the presence of a small air gap. This would have refracted photons from both the fluorescence excitation (emitted by the phone flash above the device) and fluorescence emission, potentially reducing fluorescence intensities. 